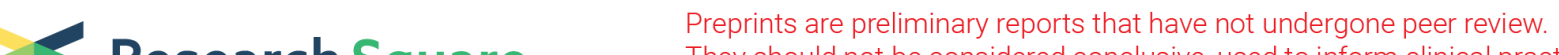 Research Square They should not be considered conclusive, used to inform clinical practice, or referenced by the media as validated information.
}

\section{Cost-utility analysis of olanzapine in four-drug antiemetic therapy in Japanese patients treated with highly emetogenic cisplatin-containing chemotherapy}

Yu Kondo ( $\nabla$ yu.kondoh.0327@gmail.com )

Toyota Kosei Hospital

Tomoya Tachi

Gifu Yakka Daigaku

Takayoshi Sakakibara

Toyota Kosei Hospital

Jun Kato

Toyota Kosei Hospital

Takahito Mizuno

Toyota Kosei Hospital

Yoshio Miyake

Toyota Kosei Hospital

Hitomi Teramachi

Gifu Yakka Daigaku

\section{Research Article}

Keywords: olanzapine, cost-effectiveness, highly emetogenic chemotherapy, cost-utility analysis

Posted Date: March 11th, 2021

DOI: https://doi.org/10.21203/rs.3.rs-232581/v1

License: (c) (i) This work is licensed under a Creative Commons Attribution 4.0 International License. Read Full License 


\section{Abstract}

Purpose Olanzapine has been shown to have an additive effect on the three-drug antiemetic therapy, consisting of aprepitant, palonosetron, and dexamethasone, in a highly emetogenic cisplatin-containing chemotherapy. Although olanzapine may be more economical than aprepitant or palonosetron, an adequate cost-efficacy analysis has not been conducted. We conducted a cost-utility analysis to evaluate the cost-effectiveness of olanzapine in four-drug antiemetic therapy among Japanese patients.

Methods We simulated model patients treated with highly emetogenic cisplatin-containing chemotherapy and developed a decision-analytical model of patients receiving triple antiemetic therapy with or without olanzapine. The cost, probabilities, and incremental cost-effectiveness ratio (ICER) of each treatment were calculated from the perspective of the Japanese healthcare payer. The threshold ICER was set at 45,867 United States dollars (USD) (5 million Japanese yen) per quality-adjusted life-year (QALY). The probabilities, utility value, and other costs were obtained from published sources. The robustness of this model was validated by one-way sensitivity analysis and probabilistic sensitivity analysis.

Results The calculated ICER was 5,156 USD/QALY, which was below the threshold. Under the set conditions, the probabilistic sensitivity analysis revealed a $100 \%$ probability that olanzapine was costeffective. Based on the one-way sensitivity analysis, reducing the cost of olanzapine below 10.78 USD placed the ICER below the threshold.

Conclusion Olanzapine was cost-effective in the four-drug antiemetic therapy for Japanese patients treated with highly emetogenic cisplatin-containing chemotherapy

\section{Introduction}

Nausea and vomiting decrease the quality of life (QOL) of patients undergoing cancer chemotherapy [1]. The control of nausea and vomiting is significant for the continuation of treatment. The guidelines of scientific societies, such as the American Society of Clinical Oncology (ASCO), the Multinational Association of Supportive Care in Cancer/European Society of Medical Oncology (MASCC/ESMO), and the National Comprehensive Cancer Network (NCCN), classify the emetogenicity of anticancer drugs into four levels [2-4]. Similarly, the Japan Society of Clinical Oncology (JSCO) guideline classifies emetogenicity into minimal ( $<10 \%)$, low $(10-30 \%)$, moderate $(30-90 \%)$, and highly emetogenic $(>90 \%)$ risks [5]. Cisplatin is one of the most emetogenic anticancer drugs, and all guidelines have classified cisplatin as highly emetogenic [2-5]. Adequate prophylactic antiemetic therapy is essential to maintain the QOL of patients taking highly emetogenic regimens.

Olanzapine inhibits multiple receptors (dopamine $\mathrm{D}_{1}, \mathrm{D}_{2}, \mathrm{D}_{3}$ receptors, serotonin 5-hydroxytryptamine type $2 \mathrm{a}\left(5-\mathrm{HT}_{2 \mathrm{a}}\right), 5-\mathrm{HT}$ type $2 \mathrm{c}\left(5-\mathrm{HT}_{2 \mathrm{c}}\right), 5-\mathrm{HT}_{3}$, and $5-\mathrm{HT}_{6}$ receptors, alpha1-adrenergic receptors, muscarinic receptors, and histamine $\mathrm{H}_{1}$ receptors) [6]. Olanzapine has been used to treat schizophrenia, but it has recently emerged as an antiemetic agent in cancer chemotherapy [7-11]. A randomized, 
double-blind, placebo-controlled phase III study reported that adding olanzapine to neurokinin-1 ( $\left.\mathrm{NK}_{1}\right)$ receptor antagonists, $5-\mathrm{HT}_{3}$ receptor antagonists, and dexamethasone brought significant benefits in preventing chemotherapy-induced nausea and vomiting (CINV) from highly emetogenic cisplatincontaining chemotherapy [7]. The ASCO guidelines recommended a four-drug combination, including a $\mathrm{NK}_{1}$-receptor antagonist, $5-\mathrm{HT}_{3}$ receptor antagonist, dexamethasone, and olanzapine, for preventing CINV after highly emetogenic chemotherapy (HEC) [2]. One of the recommended options for use in the NCCN and MASCC/ESMO guidelines was a four-drug combination that included olanzapine [3-4].

Japan has a universal health insurance system, and the Ministry of Health, Labor, and Welfare determines drug prices. The rapid aging of Japan's population and the resulting increase in medical costs have become a severe problem. Because $\mathrm{NK}_{1}$ receptor antagonists, such as aprepitant and $5-\mathrm{HT}_{3}$ receptor antagonists, are more expensive than classical antiemetics, several studies have examined their costeffectiveness in Japan and overseas [12-16]. In Japan, aprepitant use was reportedly cost-effective for outpatient treatment but not cost-effective under inpatient conditions [12]. Palonosetron, a secondgeneration $5-\mathrm{HT}_{3}$ receptor antagonist, was reportedly not cost-effective as a first-generation $5-\mathrm{HT}_{3}$ receptor antagonist [13].

On the other hand, the drug price of olanzapine per $5 \mathrm{mg}$ of is $167.4 \mathrm{JPY}$, which is lower than those of aprepitant (8,949.3 JPY/3 days) and palonosetron (14,937 JPY). Therefore, it is considered an excellent drug, not only in terms of efficacy but also in cost-effectiveness. However, the cost-effectiveness of olanzapine in antiemetic therapy has not been sufficiently investigated. Given this, this study aimed to evaluate the cost-effectiveness of olanzapine in four-drug antiemetic therapy among Japanese patients receiving highly emetogenic cisplatin-containing chemotherapy.

\section{Methods}

\section{Model}

According to previous reports, we simulated model patients treated with highly emetogenic cisplatincontaining chemotherapy and developed a decision-analytic model (Fig. 1) [12-16]. Based on a phase III clinical trial conducted in Japan (J-FORCE study) [7], the model patients received either a four-drug or a three-drug regimen. The four-drug regimen included dexamethasone (12 mg on Day 1 and $8 \mathrm{mg}$ on Days 2-4); palonosetron (0.75 mg on Day 1); aprepitant (125 mg aprepitant on Day 1, and $80 \mathrm{mg}$ on Days 2 and 3); olanzapine (5 mg on Days 1-4). The three-drug regimen consisted of the same drug regimen minus olanzapine. The costs and health state outcomes of each treatment were calculated. The model was divided into two phases: the acute phase (Day 1 ) and the delayed phase (Days 2-5). The clinical outcomes were defined as follows: complete response (CR) with no emesis and no rescue medication use, and incomplete response (IR) with some emesis or rescue medication use. CR was subdivided into two mutually exclusive health outcomes: complete protection (CP), which was defined as no emesis, rescue antiemetic therapy, and significant nausea, and complete response at best (CRB), which included 
those who achieved CR but not CP. The analysis period lasted for five days, as in previous reports [1216].

\section{Health state outcomes and probabilities}

Health state outcomes were evaluated using quality-adjusted life years (QALYS). The QALY in each treatment group was integrated according to the probability of the health state in the acute and delayed phases. A utility value for each health state was assigned to previous reports. Utility values of 0.9 for $C P$, 0.7 for CRB, and 0.2 for IR were used (Table 1$)[12,13,15,17]$. If drowsiness occurred, the utility value was assumed to decrease by 0.1 [18]. The health state probabilities and the probability of drowsiness were based on the J-FORCE study results (Table 1) [7].

Table 1

Utility values for model patient's outcomes and health state probabilities in the model

\begin{tabular}{|llll|}
\hline Parameters & Base case & \multicolumn{2}{l}{ Range for sensitivity analysis } \\
\cline { 4 - 4 } & & Lower bound & Upper bound \\
\hline Utility weight & & & \\
\hline Complete protection & 0.90 & 0.63 & 1.00 \\
\hline Complete response at best & 0.70 & 0.49 & 0.91 \\
\hline Incomplete response & 0.20 & 0.14 & 0.26 \\
\hline Drowsiness & -0.10 & -0.07 & -0.13 \\
\hline Probabilities & & & 0.90 \\
\hline CP in acute phase in olanzapine group & 0.86 & 0.82 & 0.85 \\
\hline CP in acute phase in non-olanzapine group & 0.81 & 0.77 & 0.97 \\
\hline CR in acute phase in olanzapine group & 0.95 & 0.93 & 0.92 \\
\hline CR in acute phase in non-olanzapine group & 0.89 & 0.85 & 0.71 \\
\hline CP in delayed phase in olanzapine group & 0.66 & 0.61 & 0.55 \\
\hline CP in delayed phase in non-olanzapine group & 0.50 & 0.45 & 0.83 \\
\hline CR in delayed phase in olanzapine group & 0.79 & 0.75 & 0.65 \\
\hline CR in delayed phase in non-olanzapine group & 0.60 & 0.55 & 0.51 \\
\hline Drowsiness in olanzapine group & 0.39 & 0.27 & 0.38 \\
\hline Drowsiness in non-olanzapine group & 0.29 & 0.20 & \\
\hline The probabilities are based on the J-FORCE study [7] & & & \\
\hline CP complete protection, CR complete response & & & \\
\hline
\end{tabular}




\section{Cost}

The costs of prophylactic antiemetic therapy and rescue treatments for CINV were included in the model. All costs for drugs in this study were based on the National Health Insurance Drug Price Standard listed in 2020 (Table 2). The costs calculated in JPY were converted to United States dollars (USD) using the exchange rate reported by the Organization for Economic Cooperation and Development (OECD) in 2019 (1 USD = 109.01 JPY) [19]. The rescue treatment cost was set to 7.80 USD per treatment based on the Japanese report by Tsukiyama et al. [12].

Table 2

Costs of drugs and rescue treatment

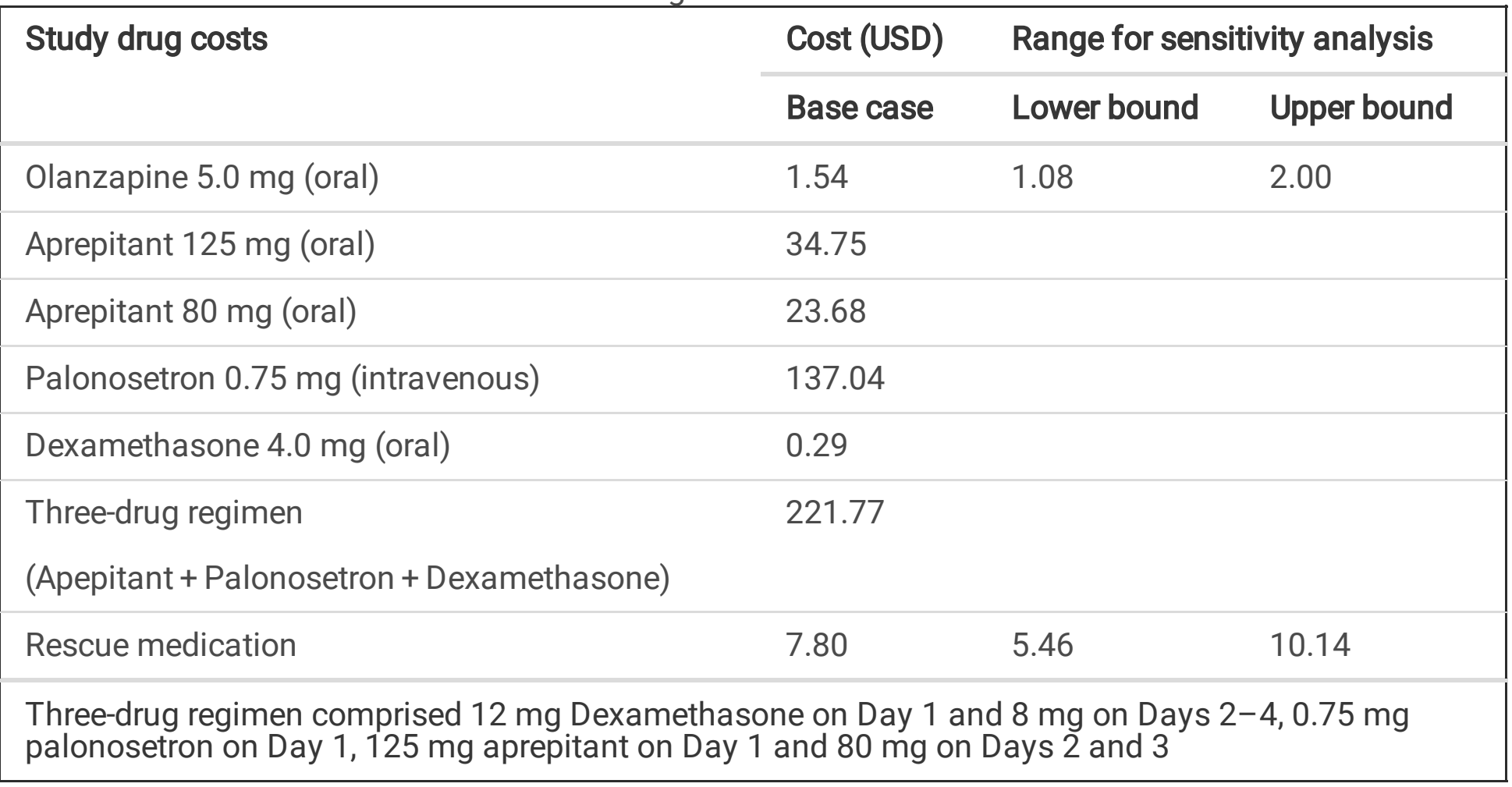

\section{Cost-utility analysis}

The incremental cost-effectiveness ratio (ICER) was calculated to evaluate the cost-effectiveness of the olanzapine-containing four-drug regimen. The ICER was calculated as follows:

ICER $($ JPY $/$ QALY $)=($ cost with olanzapine - cost without olanzapine) $/$ (QALYs with olanzapine - QALYs without olanzapine)

The willingness-to-pay (WTP) threshold was set at 45,867 USD (5,000,000 Japanese yen)/QALY defined by Shiroiwa et al. [20]. No discount was applied since the study lasted for less than a year. The cost-utility analysis was performed from the perspective of the Japanese healthcare payer. Cost-utility analyses were conducted using TreeAge® Pro 2019 (TreeAge Software Inc. Williamstown, MA, USA). 


\section{Sensitivity analysis}

The study parameters, such as drug costs, utility values, and health state probabilities, were subject to uncertainty. Therefore, by varying the parameters within a specific range, the model's uncertainty and robustness were evaluated by one-way and probabilistic sensitivity analyses. Probabilistic sensitivity analysis was conducted by performing 1,000 iterations of an automatic multiple random numbering method using Monte Carlo simulation. The drug costs, rescue treatment cost, probability of drowsiness, and utility values were varied by $\pm 30 \%$ from the base values. The health state probabilities varied within the $95 \%$ confidence interval of the J-FORCE study [7]. In the one-way sensitivity analysis, we calculated the cost of olanzapine, whose ICER was equal to WTP.

\section{Results}

\section{Base-Case analysis}

The cost was 229.92 USD in the olanzapine group and 225.26 USD in the non-olanzapine group. The QALYs gained were 0.00962 QALYs in the olanzapine group and 0.00872 QALYs in the non-olanzapine group. The incremental cost of the olanzapine group relative to the non-olanzapine group was 4.66 USD, and the incremental QALYs were 0.00090 QALYs, resulting in an ICER of 5,156 USD/QALY. This value was below the WTP, and according to the base case analysis, the olanzapine group was more cost-effective than the non-olanzapine group.

\section{Sensitivity analysis}

A tornado diagram based on the one-way sensitivity analysis is shown in Fig. 2. It is arranged in order of the degree of influence on ICER. The most influential parameter on ICER was the probability of delayed $\mathrm{CR}$ in the non-olanzapine group, followed by the cost of olanzapine (Fig. 2). In the one-way sensitivity analysis, the drug price of olanzapine with ICER equal to WTP was 10.78 USD.

The results of the probabilistic sensitivity analysis are shown in Fig. 3. Based on the scatter plot, the olanzapine group was more cost-effective than the non-olanzapine group, given that the incremental QALYs were positive below the WTP line (45,867 USD/QALY) for the points obtained by random sampling. The probabilistic sensitivity analysis revealed a $100 \%$ probability that the olanzapine group was more cost-effective than the non-olanzapine group.

\section{Discussion}

To our knowledge, this was the first report to evaluate the cost-effectiveness of olanzapine in a four-drug antiemetic regimen. In the base-case analysis, the ICER of olanzapine added to the three-drug regimen was 5,156 USD/QALY, which was lower than the set WTP. Furthermore, the probabilistic sensitivity analysis revealed a $100 \%$ probability for the cost-effectiveness of olanzapine. These results indicated that olanzapine was cost-effective in the four-drug antiemetic regimen, with a cisplatin-containing HEC regimen. Although, it is necessary to pay attention to adverse effects such as hyperglycemia and QT 
prolongation. Olanzapine was recommended, not only for its antiemetic effects, but also for its costeffectiveness.

Chanthawong et al. reported that compared to the doublet antiemetic regimen (dexamethasone plus firstgeneration $5-\mathrm{HT}_{3}$ receptor antagonist), the addition of olanzapine resulted in incremental QALY of 0.0022-0.0026, with cost savings of 2.98 USD, 27.71 USD, and 52.20 USD in Thailand, Malaysia, and Indonesia, respectively [15]. At the same time, they reported that in Singapore, switching from aprepitant to olanzapine in a triplet antiemetic regimen (dexamethasone, first-generation $5-\mathrm{HT}_{3}$ receptor antagonist, and aprepitant) resulted in cost savings of 60.91 USD and incremental QALYs of 0.0005 [15]. In Japan, there have been several reports examining the cost-effectiveness of aprepitant and palonosetron [12-14]. Kashiwa et al. reported the cost-effectiveness of palonosetron in cisplatin-containing HEC regimens based on the TRIPLE study results conducted in Japan $[13,21]$. The calculated ICER for palonosetron compared to granisetron was 16,204,591 JPY/QALY (144,465 USD/QALY). Tsukiyama et al. reported that the cost-effectiveness of aprepitant for HEC regimens was 3,906,698 JPY/QALY (35,951 USD/QALY) in the outpatient care setting and 6,195,781 JPY/QALY (56,952 USD/QALY) in the inpatient care setting [12]. The calculated ICER for olanzapine in our study was substantially lower than those obtained in the aforementioned studies because olanzapine cost less than antiemetics such as aprepitant and palonosetron. The one-way sensitivity analysis showed that the second most influential parameter on ICER was the drug price of olanzapine, and the cost of antiemetics was a factor that directly influenced ICER. In Japan, the cost of olanzapine (5 mg for four days) was 6.16 USD. Aprepitant for three days cost 82.11 USD, and single-dose palonosetron cost 137.04 USD. These indicated that the difference in drug cost per regimen had a significant impact on ICER.

This study had some limitations. First, this study only included cisplatin-based regimens. Navari et al. reported that the addition of $10 \mathrm{mg}$ of olanzapine to a three-drug regimen significantly improved acute and delayed CINV in patients who were receiving anthracycline-cyclophosphamide (AC) or cisplatin ( $\geq 70$ mg per square meter of body-surface area) [8]. However, we did not use the results of Navari et al. because severe sedation occurred in $5 \%$ of patients in their study, and there was minimal opportunity to use $10 \mathrm{mg}$ in Japan because of concerns about drowsiness. To the best of our knowledge, no placebocontrolled, randomized, or randomized trial has demonstrated the efficacy of olanzapine $5 \mathrm{mg}$ for the AC regimen. The MASCC/ESMO stated that the clinically meaningful difference was at least $10 \%$ [3]. In the JFORCE study cited in our model, the CP rate in the delayed phase was $60 \%$ in the olanzapine group and $50 \%$ in the non-olanzapine group [7]. If the same efficacy level is demonstrated in the AC regimen, olanzapine will be highly cost-effective in the AC regimen. Second, the approved palonosetron dose in Japan is $0.75 \mathrm{mg}$, which was different from other countries. ASCO, NCCN, and MASCC/ESMO guidelines recommended $0.25 \mathrm{mg}$ intravenous and $0.5 \mathrm{mg}$ oral palonosetron [2-4]. The dosage of palonosetron in Japan was set to $0.75 \mathrm{mg}$ because a domestic phase II study on moderately emetogenic chemotherapy showed that this dosage had a higher delayed phase CR rate than $0.25 \mathrm{mg}$ [22]. Although, no statistically significant difference was observed. However, since the Navari et al. report included $75 \%$ of patients using $0.25 \mathrm{mg}$ of palonosetron as a $5-\mathrm{HT}_{3}$ receptor antagonist, the difference in palonosetron dose may have 
had a small effect on the results of this study. Third, this study did not take into account additional hospitalization, laboratory, and consultation costs. However, when these costs were included in the analysis, they added additional costs to the non-olanzapine group, further favoring the olanzapine group. Therefore, the omission of these costs did not affect the results and robustness of this study.

In conclusion, olanzapine was highly cost-effective in highly emetogenic cisplatin-containing risk regimens. Therefore, the use of a four-drug regimen, including olanzapine, was recommended in terms of cost-effectiveness.

\section{Declarations}

Funding: No funding was received.

Conflicts of interest/Competing interests: The authors have no conflicts of interest or competing interests to declare that are relevant to the content of this article.

Availability of data and material: The authors have full control of the data, and data is available upon request.

Code availability: Analyses were performed with TreeAge ${ }^{\circledR}$ Pro 2019 (TreeAge Software Inc. Williamstown, MA, USA).

Authors' contributions: All authors were involved in the design of the study. YK and HT coordinated the study. YK, TT, and HT drafted the manuscript. YK, TS, JK, TM, and YM performed the data analyses. All authors critically revised the manuscript and read and approved the final manuscript.

Ethics approval: Not applicable.

Consent to participate: Not applicable.

Consent for publication: All authors consented to publish this paper.

\section{References}

1. Bloechl-Daum B, Deuson RR, Mavros P et al (2006) Delayed nausea and vomiting continue to reduce patients' quality of life after highly and moderately emetogenic chemotherapy despite antiemetic treatment. J Clin Oncol 24:4472-4478

2. Hesketh PJ, Kris MG, Basch E et al (2020) Antiemetics: Guideline Update. J Clin Oncol 38:2782-2797

3. Rolia F, Molassiotis A, Herrstedt J et al (2016) 2016 MASCC and ESMO guideline update for the prevention of chemotherapy- and radiotherapy-induced nausea and vomiting and of nausea and vomiting in advanced cancer patients. Ann Oncol 27(suppl 5):v119-v133. 
4. NCCN practice guidelines in oncology. Antiemesis 2020 ver. 2 (2020). [Cited 23 April 2020.] Available from URL: https://www.nccn.org/professionals/physician_gls/pdf/antiemesis.pdf

5. Aogi K, Takeuchi H, Saeki T et al (2020) Optimizing antiemetic treatment for chemotherapy-induced nausea and vomiting in Japan: Update summary of the 2015 Japan Society of Clinical Oncology Clinical Practice Guidelines for Antiemesis. Int J Clin Oncol. https://doi.org/10.1007/s10147-02001818-3

6. Bymaster FP, Calligaro DO, Falcone JF et al (1996) Radioreceptor binding profile of the atypical antipsychotic olanzapine. Neuropsychopharmacology 14:87-96

7. Hashimoto $\mathrm{H}$, Abe M, Tokuyama $\mathrm{O}$ et al (2020) Olanzapine $5 \mathrm{mg}$ plus standard antiemetic therapy for the prevention of chemotherapy-induced nausea and vomiting (J-FORCE): a multicentre, randomised, double-blind, placebo-controlled, phase 3 trial. Lancet Oncol 21:242-249

8. Navari RM, Gray SE, Kerr AC et al (2016) Olanzapine for the prevention of chemotherapy-induced nausea and vomiting. N Engl J Med 375:134-142

9. Clemmons AB, Orr J, Andrick B et al (2018) Randomized, placebo-controlled, phase III trial of fosaprepitant, ondansetron, dexamethasone (FOND) versus FOND Plus olanzapine (FOND-0) for the prevention of chemotherapy-induced nausea and vomiting in patients with hematologic malignancies receiving highly emetogenic chemotherapy and hematopoietic cell transplantation regimens: the FOND-0 trial. Biol Blood Marrow Transplant 24:2065-2071

10. Tienchaiananda P, Nipondhkit W, Maneenil K et al (2019) A randomized, double-blind, placebocontrolled study evaluating the efficacy of combination olanzapine, ondansetron and dexamethasone for prevention of chemotherapy-induced nausea and vomiting in patients receiving doxorubicin plus cyclophosphamide. Ann Palliat Med 8:372-380

11. Navari RM, Gray SE, Kerr AC (2011) Olanzapine versus aprepitant for the prevention of chemotherapy-induced nausea and vomiting: A randomized phase III trial. J Support Oncol 9:188195

12. Tsukiyama I, Hasegawa S, Ikeda Y et al (2018) Cost-effectiveness of aprepitant in Japanese patients treated with cisplatin-containing highly emetogenic chemotherapy. Cancer Science 109:2881-2888

13. Kashiwa M, Matsusita R (2019) Cost-utility analysis of palonosetron in the antiemetic regimen for cisplatin-containing highly emetogenic chemotherapy in Japan BMC Health Serv Res. https://doi.org/10.1186/s12913-019-4281-0

14. Tsukiyama I, Ando M, Tsukiyama S (2019) Cost-utility analysis of aprepitant for patients who truly need it in Japan. Support Care Cancer 27:3749-3758

15. Chanthawong S, Lim YH, Subongkot S et al (2019) Cost-effectiveness analysis of olanzapinecontaining antiemetic therapy for managing highly emetogenic chemotherapy in Southeast Asia: a multinational study. Support Care Cancer 27:1109-1119

16. Humphreys S, Pellissier J, Jones A (2013) Cost-effectiveness of an aprepitant regimen for prevention of chemotherapy-induced nausea and vomiting in patients with breast cancer in the UK Cancer Manag Res. https://doi.org/10.2147/CMAR.S44539 
17. Botteman M, Nickel K, Corman S et al (2020) Cost-effectiveness of a fixed combination of netupitant and palonosetron (NEPA) relative to aprepitant plus granisetron (APR + GRAN) for prophylaxis of chemotherapy-induced nausea and vomiting (CINV): a trial-based analysis. Support Care Cancer 28:857-866

18. Neighbors DM, Bell TJ, Wilson J et al (2001) Economic evaluation of the fentanyl transdermal system for the treatment of chronic moderate to severe pain. J Pain Symptom Manage 21:129-143

19. OECD (2020), Exchange rates (indicator). https://data.oecd.org/conversion/exchange-rates.htm. Accessed 26 December 2020

20. Shiroiwa T, Sung YK, Fukuda T et al (2010) International survey on willingness-to-pay (WTP) for one additional QALY gained: what is the threshold of cost effectiveness? Health Econ 19:422-437

21. Suzuki K, Yamanaka T, Hashimoto $\mathrm{H}$ et al (2016) Randomized, double-blind, phase III trial of palonosetron versus granisetron in the triplet regimen for preventing chemotherapy-induced nausea and vomiting after highly emetogenic chemotherapy: TRIPLE study. Ann Oncol 27:1601-1606

22. Segawa Y, Aogi K, Inoue K et al (2009) A phase II dose-ranging study of palonosetron in Japanese patients receiving moderately emetogenic chemotherapy, including anthracycline and cyclophosphamide-based chemotherapy. Ann Oncol 20:1874-1880

\section{Figures}
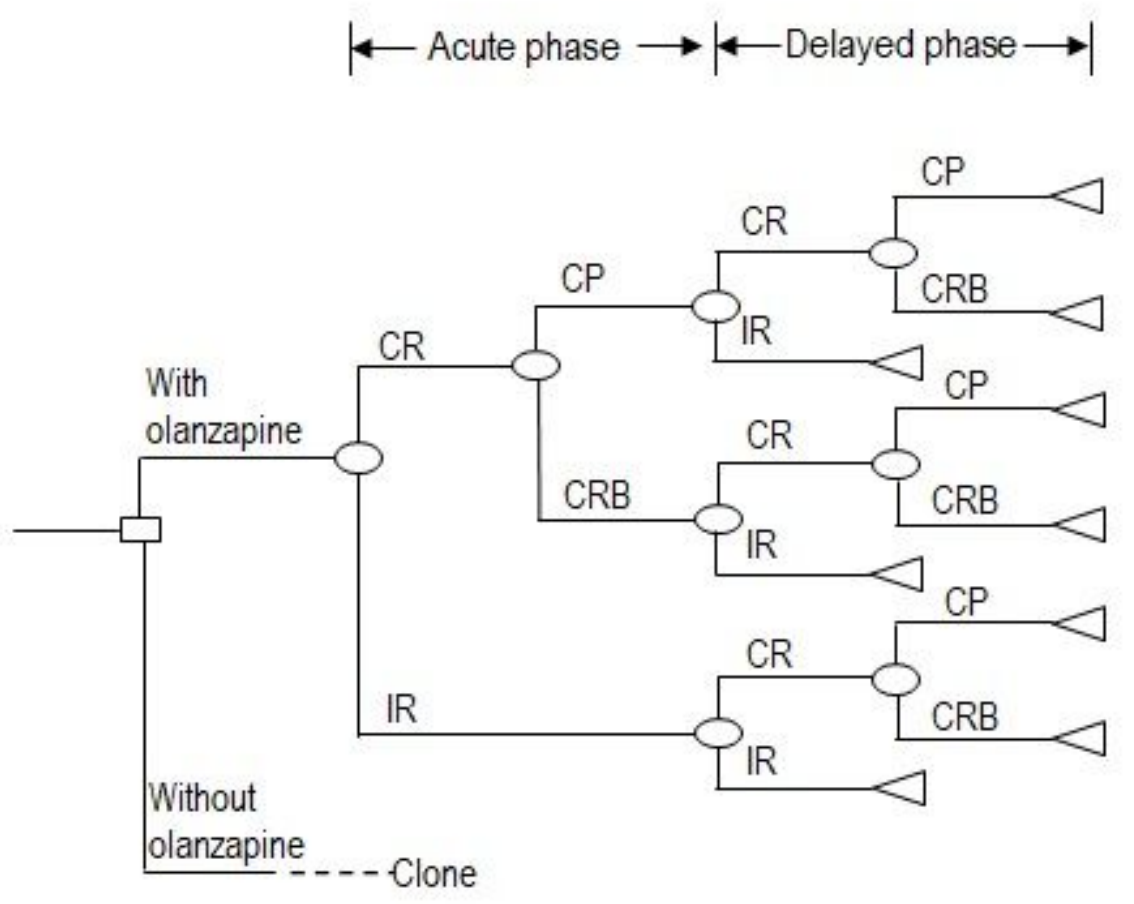

\section{Figure 1}


Decision-analytic model for cost-utility analysis. The decision tree shows the nine possible health states that a model patient can experience after receiving an antiemetic regimen. Olanzapine-containing regimen comprised dexamethasone, palonosetron, aprepitant, and olanzapine. $\mathrm{CP}$, complete protection; $\mathrm{CRB}$, complete protection at best; IR, incomplete response.

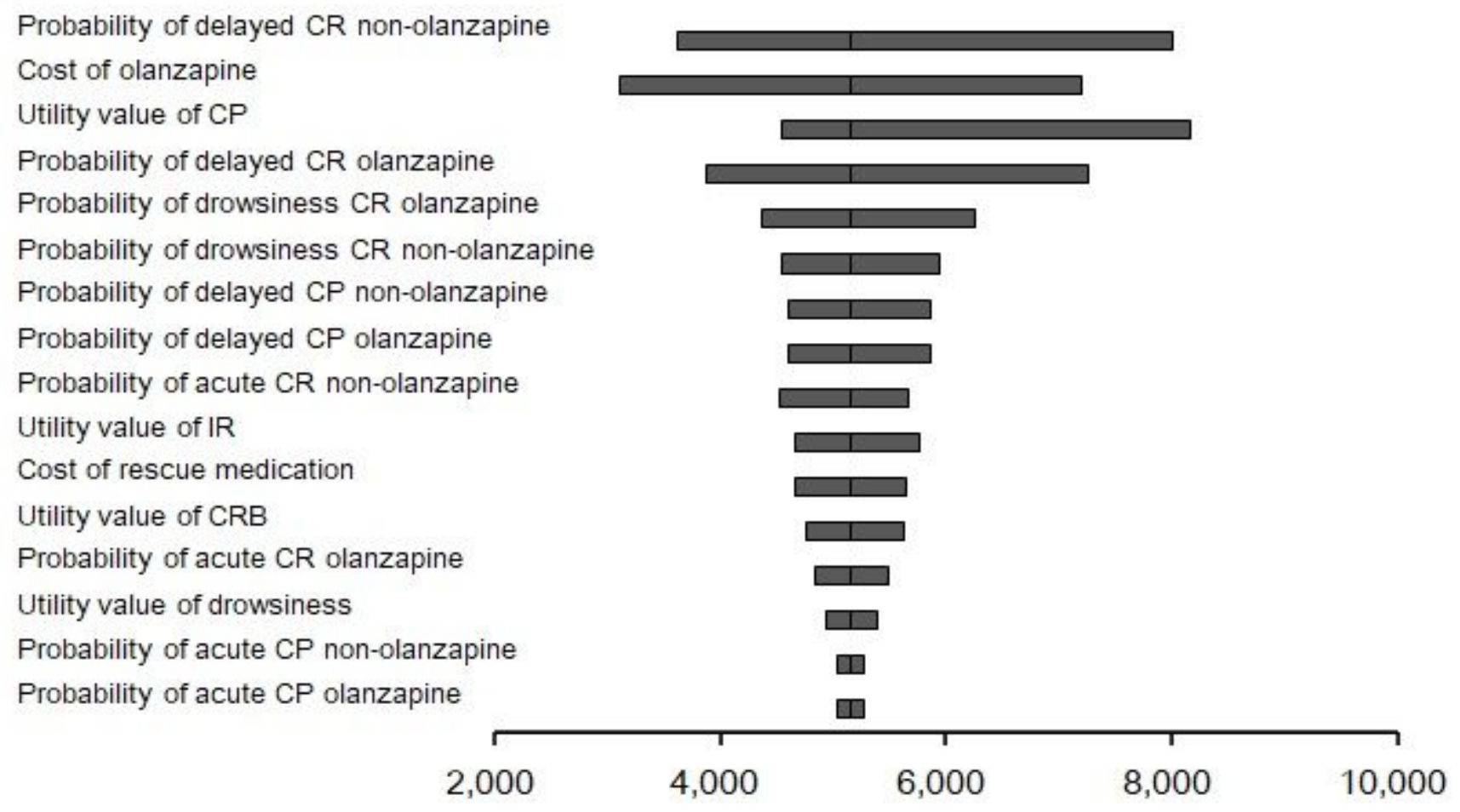

Figure 2

The result of one-way sensitivity analysis. $\mathrm{CR}$, complete response; $\mathrm{CP}$, complete protection; IR, incomplete response; CRB, complete response at best

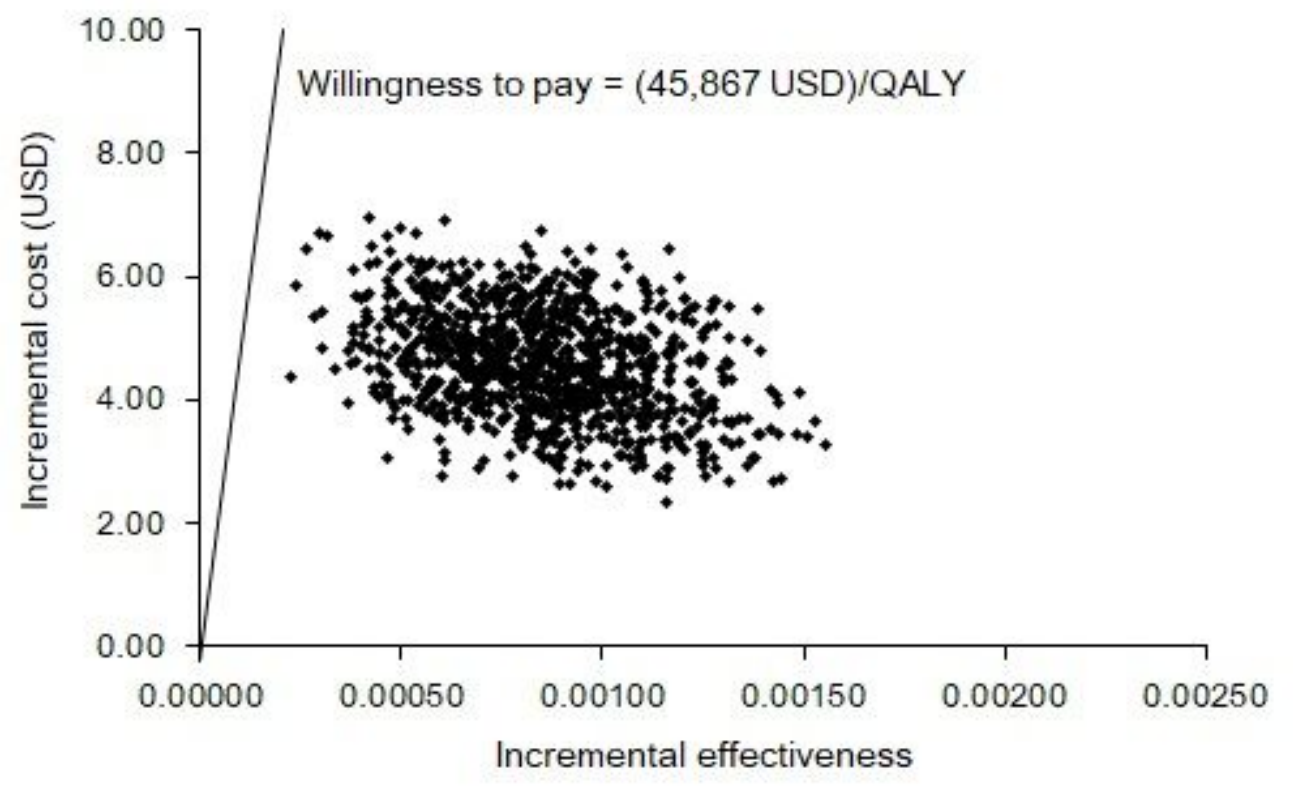

Figure 3 
The result of probabilistic analysis by scatter plot. USD, United States dollar 